\title{
PERTURBATIONS OF KANTOWSKI-SACHS MODELS
}

\author{
MICHAEL BRADLEY * and MATS FORSBERG \\ Department of Physics, Umeå University, Sweden \\ *E-mail: michael.bradley@physics.umu.se \\ ZOLTÁN KERESZTES and LÁSZLÓ Á. GERGELY \\ Departments of Theoretical Physics and Experimental Physics, University of Szeged, Hungary \\ PETER K.S. DUNSBY \\ Department of Mathematics and Applied Mathematics, University of Cape Town, South Africa
}

\begin{abstract}
Perturbations of Kantowski-Sachs models with a positive cosmological constant are considered in a harmonic decomposition, in the framework of gauge invariant $1+3$ and $1+1+2$ covariant splits of spacetime. Scalar, vector and tensor modes are allowed, however they remain vorticity-free and of perfect fluid type. The dynamics is encompassed in six evolution equations for six harmonic coefficients.
\end{abstract}

\section{Introduction}

We investigate perturbations of Kantowski-Sachs models with a positive cosmological constant. Although a simple argument by Börner and Ehlers ${ }^{1}$ renders isotropic bouncing universes incompatible with observations, the argument does not hold for Kantowski-Sachs models, ${ }^{2}$ in some of which the universe exhibits an anisotropic bounce from a contracting into an expanding phase. Hence the evolution and propagation of perturbations in these models, also their possible effects on observables, like the Sachs-Wolfe effect ${ }^{3}$ are of interest.

The $1+3$ and $1+1+2$ covariant splits of the space-time ${ }^{4} 7$ prove particularly suitable for perturbation theory, as they employ variables vanishing on the background, hence with gauge invariant perturbations. ${ }^{[}$For simplicity we consider only vorticity-free perturbations of perfect fluid type, but allow for scalar, vector and tensor modes. We further expand the evolution equations for the perturbative variables in terms of harmonics.

\section{The $1+3$ and $1+1+2$ covariant formalisms}

The existence of a preferred timelike vector $u^{a}$ naturally leads to a covariant $1+3$ split of spacetimes $\frac{677}{6}$ The induced metric $h_{a}^{b}=g_{a}^{b}+u_{a} u^{b}$ also acts as a projection operator onto the perpendicular 3-space. All vectors and tensors can be covariantly decomposed into "spatial" and "timelike" parts. Covariant time derivative and projected spatial derivative can be defined as

$$
\dot{\psi}_{a . . b} \equiv u^{c} \nabla_{c} \psi_{a \ldots b} \quad \text { and } \quad D_{c} \psi_{a \ldots b} \equiv h_{c}^{f} h_{a}^{d} \ldots h_{b}^{e} \nabla_{f} \psi_{d \ldots e}
$$

respectively. The covariant derivative of the 4 -velocity $u^{a}$ is decomposed as

$$
\nabla_{a} u_{b}=-u_{a} A_{b}+D_{a} u_{b}=-u_{a} A_{b}+\frac{1}{3} \theta h_{a b}+\omega_{a b}+\sigma_{a b}
$$


in terms of the kinematic quantities of $u^{a}$, the acceleration $A_{a} \equiv u^{b} \nabla_{b} u_{a}$, expansion $\theta \equiv D_{a} u^{a}$, vorticity $\omega_{a b} \equiv D_{[a} u_{b]}$ and shear $\sigma_{a b} \equiv D_{<a} u_{b>}$ respectively. The kinematic quantities, supplemented by the Ricci tensor (expressed via the Einstein equations in terms of the energy density $\mu$ and pressure $p$ of a perfect fluid) and by the electric and magnetic parts of the Weyl tensor $\left(E_{a b} \equiv C_{a c b d} u^{c} u^{d}\right.$ and $\left.H_{a b} \equiv \frac{1}{2} \eta_{a d e} C_{b c}^{d e} u^{c}\right)$ are the dependent variables. The Ricci and Bianchi identities lead to both evolution equations in the $u^{a}$ direction and to constraints.

A formalism for a further split $(1+2)$ with respect to a spatial vector $n^{a}$ (with $\left.u^{a} n_{a}=0\right)$ is also known. $\frac{415}{}$ The projections perpendicular to $n^{a}$ arise via the induced metric $N_{a}^{b}=h_{a}^{b}-n_{a} n^{b}$ of the 2-space. Vectors and tensors may be decomposed again into scalars along $n^{a}$, perpendicular two-vectors and symmetric, trace-free two-tensors as $A^{a}=\mathcal{A} n^{a}+\mathcal{A}^{a}, \omega^{a}=\Omega n^{a}+\Omega^{a}, \sigma_{a b}=\Sigma\left(n^{a} n^{b}-\frac{1}{2} N_{a b}\right)+2 \Sigma_{(a} n_{b)}+\Sigma_{a b}$ and similarly for $E_{a b}$ and $H_{a b}$ in terms of $\mathcal{E}, \mathcal{E}_{a}, \mathcal{E}_{a b}$ and $\mathcal{H}, \mathcal{H}_{a}, \mathcal{H}_{a b}$ respectively. The derivatives along and perpendicular to $n^{a}$ are

$$
\hat{\psi}_{a \ldots b} \equiv n^{c} D_{c} \psi_{a \ldots b}=n^{c} h_{c}^{f} h_{a}^{d} \ldots h_{b}^{e} \nabla_{f} \psi_{d \ldots e} \text { and } \delta_{c} \psi_{a \ldots b} \equiv N_{c}^{f} N_{a}^{d} \ldots N_{b}^{e} D_{f} \psi_{d \ldots e}
$$

respectively. Similarly to $\nabla_{a} u_{b}$, the derivatives $D_{a} n_{b}$ and $\dot{n}_{a}$ can be decomposed in terms of 'kinematical' quantities related to $n^{a}$ as

$$
D_{a} n_{b}=n_{a} a_{b}+\frac{1}{2} \phi N_{a b}+\xi \epsilon_{a b}+\zeta_{a b} \quad \text { and } \quad \dot{n}_{a}=\mathcal{A} u_{a}+\alpha_{a}
$$

where $a_{a} \equiv \hat{n}_{a}, \phi \equiv \delta_{a} n^{a}, \xi \equiv \frac{1}{2} \epsilon^{c a b d} \delta_{a} n_{b} u_{c} n_{d}, \zeta_{a b} \equiv \delta_{\{a} n_{b\}}, \mathcal{A} \equiv n^{a} A_{a}, \alpha_{a} \equiv N_{a}^{b} \dot{n}_{b}$.

As summary, the Ricci and Bianchi identities can be rewritten as evolution and propagation equations in the $u^{a}$ and $n^{a}$ directions plus constraints.

\section{Perturbations of Kantowski-Sachs}

For the unperturbed space-time we take Locally Rotationally Symmetric (LRS) Kantowski-Sachs cosmologies 9

$$
d s^{2}=-d t^{2}+a_{1}^{2}(t) d z^{2}+a_{2}^{2}(t)\left(d \vartheta^{2}+\sin ^{2} \theta d \varphi^{2}\right)
$$

with cosmological constant $\Lambda>0$ and a perfect fluid with barytropic equation of state $p=p(\mu)$. These space-times are completely determined by the shear $\Sigma$, energy density $\mu$, expansion $\theta$. The electric part of the Weyl tensor $\mathcal{E}$ is given algebraically in terms of the others.

The perturbative analysis proceeds smoothly in terms of variables vanishing on the background, hence being gauge invariant ${ }^{[}$The spatial variations in $\theta, \Sigma, \mathcal{E}, \mu$ are characterized by their gradients

$$
W_{a} \equiv \delta_{a} \theta, \quad V_{a} \equiv \delta_{a} \Sigma, \quad X_{a} \equiv \delta_{a} \mathcal{E}, \quad \mu_{a} \equiv \delta_{a} \mu,
$$

vanishing on the background (the derivatives $\hat{\theta} \equiv n^{a} D_{a} \theta$ etc. can be rewritten in terms of $\delta_{a}$-derivatives due to the commutation relations holding in the case of no vorticity). Other variables vanishing on the background are $a_{a}, \phi, \xi, \zeta_{a b}, \alpha_{a}, \mathcal{A}, \mathcal{A}_{a}$, $\Sigma_{a}, \Sigma_{a b}, \mathcal{E}_{a}, \mathcal{E}_{a b}, \mathcal{H}, \mathcal{H}_{a}, \mathcal{H}_{a b}$, where $a_{a}$ can be put to zero by choice of frame. 
Any scalar, vector and tensor variable is expanded in harmonics according to

$$
\begin{aligned}
\Psi & =\sum_{k_{\|}, k_{\perp}} \Psi_{k_{\|} k_{\perp}} P_{k_{\|}} Q_{k_{\perp}}, \quad \Psi_{a}=\sum_{k_{\|}, k_{\perp}} P_{k_{\|}}\left(\Psi_{k_{\|} k_{\perp}}^{V} Q_{a}^{k_{\perp}}+\bar{\Psi}_{k_{\|} k_{\perp}}^{V} \bar{Q}_{a}^{k_{\perp}}\right), \\
\Psi_{a b} & =\sum_{k_{\|}, k_{\perp}} P_{k_{\|}}\left(\Psi_{k_{\|}, k_{\perp}}^{T} Q_{a b}^{k_{\perp}}+\bar{\Psi}_{k_{\|}, k_{\perp}}^{T} \bar{Q}_{a b}^{k_{\perp}}\right)
\end{aligned}
$$

where $Q_{k_{\perp}}, Q_{a}^{k_{\perp}}, \bar{Q}_{a}^{k_{\perp}}, Q_{a b}^{k_{\perp}}$ and $\bar{Q}_{a b}^{k_{\perp}}$ are harmonics on the 2-spheres of constant $z$ and $P_{k_{\|}}$are the corresponding expansion functions in the $z$-direction.

All first order quantities can be given in terms of six coefficients. Four of them, $\mu_{k_{\|}, k_{\perp}}^{V}, \Sigma_{k_{\|}, k_{\perp}}^{T}, \mathcal{E}_{k_{\|}, k_{\perp}}^{T}$ and $\overline{\mathcal{H}}_{k_{\|}, k_{\perp}}^{T}$ form a closed first order system of evolution equations coupled to the density gradient, in agreement with earlier results for scalar perturbations. ${ }^{[2]}$ The two other $\overline{\mathcal{E}}_{k_{\|}, k_{\perp}}^{T}$ and $\mathcal{H}_{k_{\|}, k_{\perp}}^{T}$ form a closed system for free waves.

These sets of equations seem suitable to study the propagation of gravitational waves, and the coupling between scalar and tensor perturbations. Furthermore, the null geodesic condition for photons leads to equations giving the redshift in different directions in terms of the $1+1+2$ quantities. Hence the Sachs-Wolfe effect and the corresponding variations in the CMB temperature can be calculated.

\section{Acknowledgements}

Zoltán Keresztes was supported by OTKA grant no. 100216

\section{References}

1. G. Börner and J. Ehlers, Astron. Astrophys., 204, 1 (1988)

2. M. Bradley, P. K. S. Dunsby, M. Forsberg, and Z. Keresztes, Class. Quantum Grav. 29, 095023 (2012).

3. R. K. Sachs, and A. M. Wolfe, Astrophys. J. 147, 73 (1967)

4. C.A. Clarkson, Phys. Rev. D, 76, 104034 (2007).

5. C. A. Clarkson and R. Barrett, Class. Quantum Grav. 20, 3855 (2003).

6. G.F.R Ellis and M. Bruni, Phys. Rev. D, 40, 1804 (1989).

7. G.F.R Ellis and H. van Elst, in M. Lachieze-Rey (ed.), Theoretical and Observational Cosmology, NATO Science Series, Kluwer Academic Publishers (1998), arXiv:gr-qc/9812046v5.

8. J.M. Stewart and M. Walker, Proc. R. Soc. London, A341, 49 (1974).

9. R. Kantowski and R.K. Sachs, J. Math. Phys. 7, 443 (1967). 